\title{
Enthalpy-Entropy Compensation in the Gelatinization of Cyperus Starch
}

\section{Cyperus Nişastasının Jelatinizasyonunda Entalpi-Entropi Kompensasyonu}

\author{
(D) Jonghoon KANG1*, (D) Luz GREEN², (D) Kwajalen HALL³ \\ 1Department of Biology, Valdosta State University, Valdosta, Georgia, USA \\ 2Department of Psychology, Valdosta State University, Valdosta, Georgia, USA \\ 3Department of Chemistry, Valdosta State University, Valdosta, Georgia, USA
}

Key words: Enthalpy, entropy, thermodynamics, starch, gelatinization

Anahtar kelimeler: Entalpi, entropi, termodinamik, nişasta, jelatinizasyon

\section{Dear Editor,}

A recent paper by Olayemi et al.' examined various properties of starch cross-linked with citric acid to improve its physicochemical characteristics. This modified starch has considerable applications in food and pharmaceutical industries. The citric acid used in the study was derived from fruit wastes (orange peels)-an approach followed in green chemistry. We agree with the authors that the green chemistry approach is valuable from an environmental perspective because of the various benefits it confers, such as safe operability, faster processing, easy scalability, and less energy consumption. ${ }^{2}$

Of the physicochemical characteristics of starch, the authors measured the thermodynamic parameters of gelatinization. These parameters are used to examine the effects of sampling time point of the liquid substrates of orange peels, which served as a source of citric acid for starch modification. The authors prepared four types of starch: TSO, TS1, TS2, and TS3. TSO is native starch without any cross-linking, whereas TS1, TS2, and TS3 are starch types cross-linked with citric acid in the liquid substrates of orange peels, which were produced by natural solid-state fermentation on day 5,10 , and 15 , respectively. The authors performed differential scanning calorimetry to characterize the gelatinization of the four sets of starch in terms of thermodynamics. They measured enthalpy $(\Delta H)$ and three characteristic temperatures, onset, peak, and conclusion, of gelatinization.

On the basis of their data, the value of $\Delta H$ was positive for all four samples, indicating that starch gelatinization is an endergonic process. Although $\Delta H$ provides valuable information about starch gelatinization, another major thermodynamic parameter, entropy $(\Delta S)$, was not discussed in their paper. In this letter, we present our analysis of their results to provide the numerical values of $\Delta S$ in the gelatinization of various types of starch.

Starch gelatinization is a type of phase transition, ${ }^{3}$ and $\Delta S$ in a phase transition can be determined by the following equation: ${ }^{4}$

$$
\Delta S=\frac{\Delta H}{T_{m}}
$$

equation

Where $T_{m}$ is the melting temperature, the mid-point temperature in phase transition. ${ }^{3}$ Although information about $T_{m}$ was not available in the original paper, the peak temperature in starch gelatinization was found to be in considerable agreement with $T_{m}{ }^{3}$ Therefore, we used the peak temperature reported in the original paper as a substitute for $T_{m}$ in our analysis. Using the numerical values of $\Delta H$ and the peak temperature in the gelatinization of the four types of starch in equation (1), we obtained $\Delta S$ for each type.

\footnotetext{
*Correspondence: jkang@valdosta.edu, Phone: 1-229-333-7140, ORCID-ID: orcid.org/0000-0001-5594-6141

Received: 26.04.2021, Accepted: 05.05.2021

๑Turk J Pharm Sci, Published by Galenos Publishing House.
} 
Figure 1 shows the resulting values of $\Delta S$ and $\Delta H$ for each type of starch. The graph clearly illustrates two features of starch gelatinization. First, both native and cross-linked types of starch had positive values of $\Delta S$. This implies that starch gelatinization is an entropy-driven process $(\Delta S>0)$, regardless of the modification status. Second, $\Delta H$ exhibited a wide variation among the starch types. For instance, the difference of $\Delta H$ between TS1 and TS2 was $>1500 \mathrm{~J} / \mathrm{g}$; however, the difference in their peak temperature was $\left\langle 20^{\circ} \mathrm{C}\right.$. This is a consequence of the compensatory behavior between enthalpy and entropy, which is referred to as enthalpy-entropy compensation. ${ }^{5}$ Linear regression identified a numerical relationship between enthalpy and entropy ( $R^{2}$ : 0.9982):

$$
\Delta S=\alpha \times \Delta H+\beta
$$

equation

Where the values of $\alpha$ and $\beta$ and their standard errors were $2.4 \times 10^{-3} \pm 7.3 \times 10^{-5}$ and $4.7 \times 10^{-2} \pm 0.11$, respectively.

This phenomenon has been observed in various weakly coupled systems such as the melting of human hair, which is made of keratin proteins. ${ }^{5}$ Our analysis elucidated that modification of starch through cross-linking with citric acid can result in a significant variation in both $\Delta H$ and $\Delta S$ in gelatinization (Figure 1). However, owing to the enthalpy-entropy compensation, the variation in the peak temperature was highly limited to $17^{\circ} \mathrm{C}$.

Another feature evident in the results of the original paper ${ }^{1}$ is the inconsistency between the duration of fermentation of orange peels and the thermodynamic parameters (Figure 2). Although the authors proposed a plausible mechanism for the inconsistency, it clearly demonstrated a unique feature of green chemistry: minimum human intervention on a system results in unpredictable fluctuations in the physical and chemical variables of the system. One of the chemical variables of the orange peel substrates examined in the original paper ${ }^{1}$ was the $\mathrm{pH}$ of the orange peel liquid substrates. Although the $\mathrm{pH}$ itself

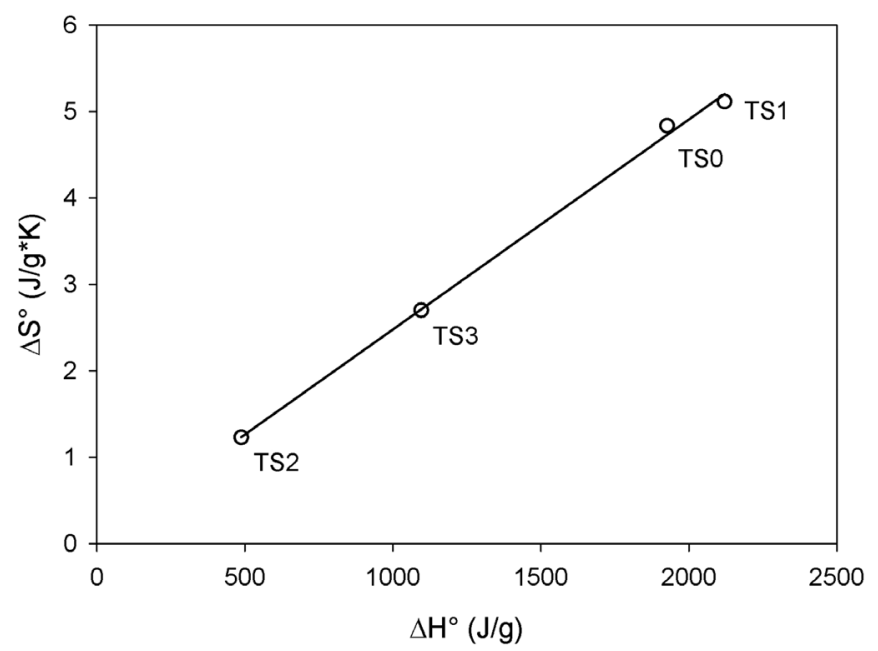

Figure 1. Enthalpy-entropy compensation in starch gelatinization. Solid line is a plot of equation (2). Linear regression was performed using SigmaPlot (Systat Software, San Jose, CA) was inconsistent with fermentation duration (Figure 2), we recognized a significant correlation between the thermodynamic parameters of gelatinization and $\mathrm{pH}$ (Figure 3 ) as the $\mathrm{R}^{2}$ value in both cases was 0.9951 . The correlation of enthalpy and entropy with $\mathrm{pH}$ is shown in equations (3) and (4), respectively:

$$
\Delta H=a_{1} \exp \left(-b_{1} \times p H\right) \quad \text { equation }
$$

$\Delta S=a_{2} \exp \left(-b_{2} \times p H\right)$

equation

Where $\quad a_{1}=11.6 \times 10^{4} \pm 4.6 \times 10^{4}, \quad b_{1}=2.0 \pm 0.2, \quad a_{2}=243.9 \pm 92.5$, and $b_{2}=1.9 \pm 0.2$. Equations (3) and (4) together elucidate a mathematical reason for the enthalpy-entropy compensation in starch gelatinization (Figure 1). Normalized enthalpy and entropy showed a similar $\mathrm{pH}$ dependency: The decreasing factors were 2 and 1.9 for enthalpy and entropy, respectively. The t-test indicated that the difference between $b_{1}$ and $b_{2}$
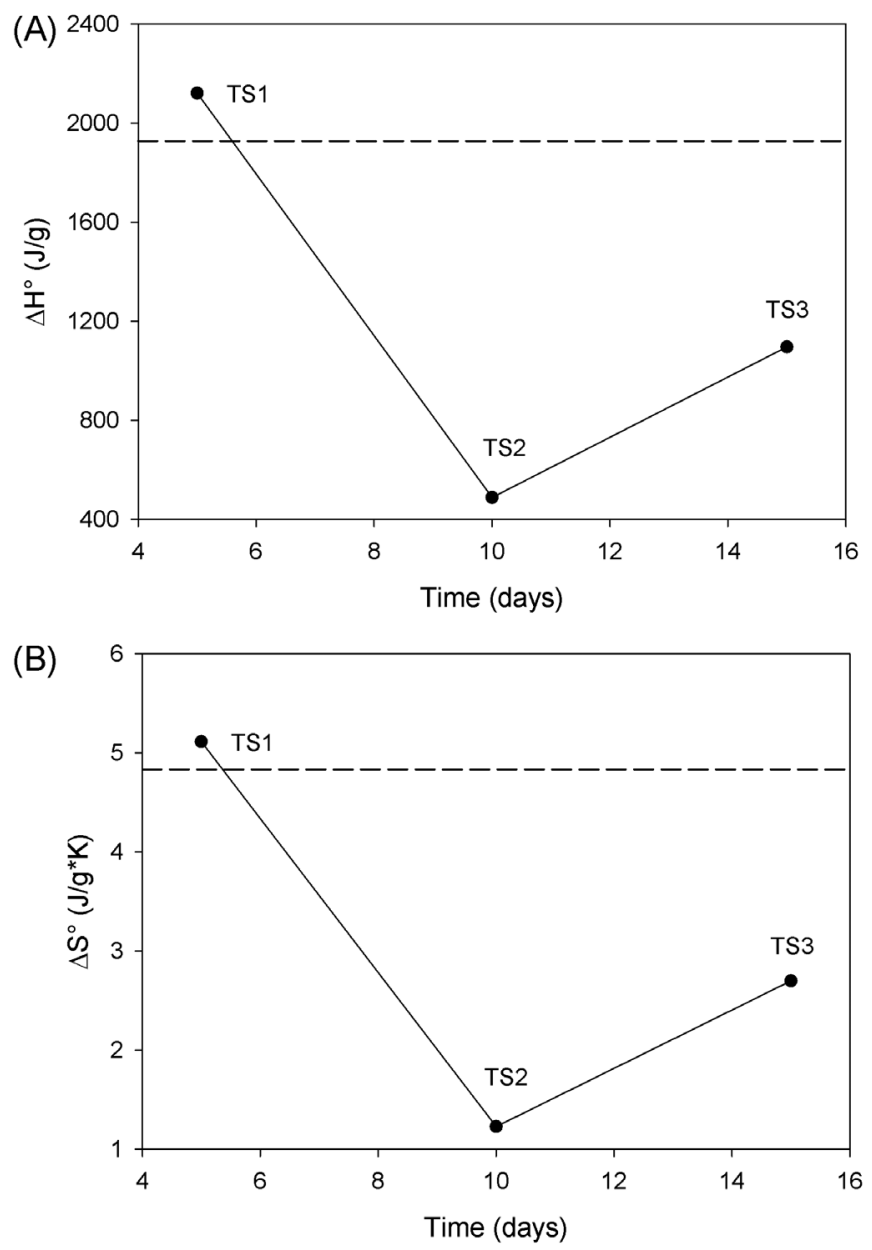

Figure 2. Correlation of enthalpy (A) and entropy (B) with time. The correlation coefficient between enthalpy and time was $-0.621 \quad(p=0.573)$ and between entropy and time was $-0.615(p=0.578)$. In both cases, no correlation was noted between any pairs of the parameters shown in the figures as $p$ was $>0.05$. The dashed line in each plot represents the corresponding value of the native starch (TSO) 


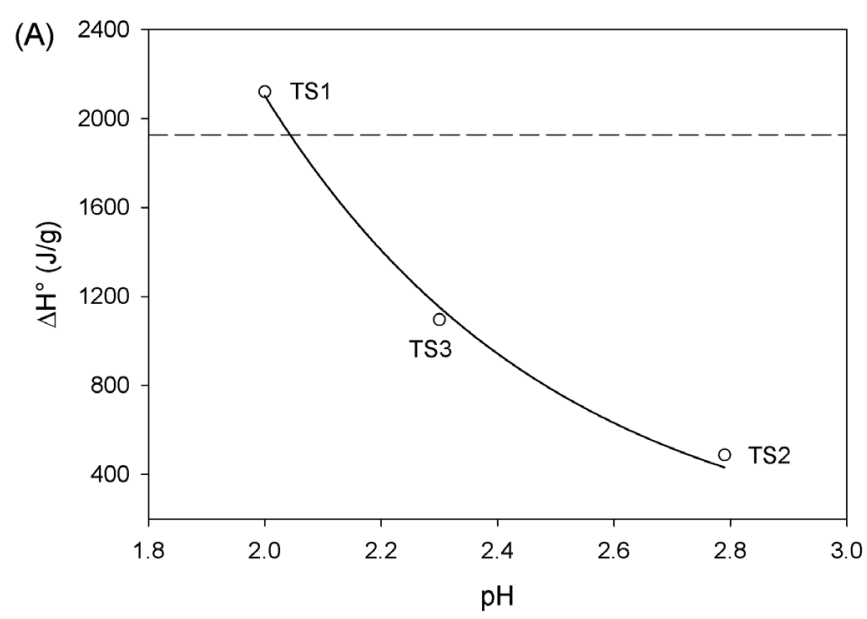

(B)

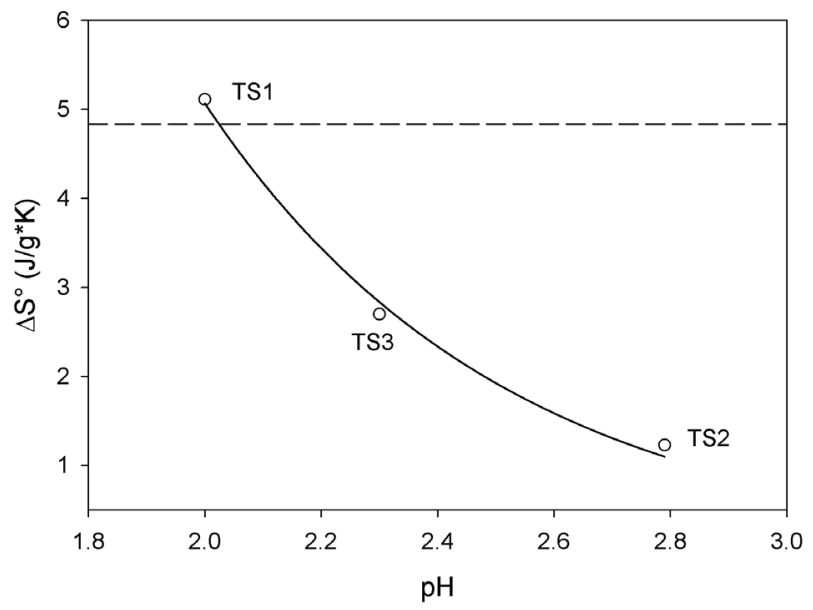

Figure 3. Correlation of $\mathrm{pH}$ with enthalpy (A) and entropy (B). Solid lines are the best fits of each case: Equation (3) in (A) and equation (4) in (B). The dashed line in each plot represents the value of the native starch (TSO)

was not statistically significant (t: 0.254, df: 2, $p=0.823$ ), strongly suggesting that enthalpy and entropy have a similar $\mathrm{pH}$ dependency. This numerical similarity is responsible for the high degree of correlation between enthalpy and entropy (Figure 1).

In conclusion, cross-linking of starch with citric acid at various $\mathrm{pH}$ values shows an enthalpy-entropy compensation. This compensation may be attributed to the similar quantitative relationship of $\mathrm{pH}$ with enthalpy and entropy. Finally, we would like to correct an error in the original paper that the unit of enthalpy of gelatinization is $\mathrm{J} / \mathrm{g}$ and not $\mathrm{J} / \mathrm{g}^{*} \mathrm{~K}$.

\section{ACKNOWLEDGMENTS}

Jonghoon KANG is grateful to James Nienow of the Department of Biology at Valdosta State University for insightful discussions.

Conflict of interest: No conflict of interest was declared by the authors. The authors are solely responsible for the content and writing of this paper.

\section{REFERENCES}

1. Olayemi B, Isimi CY, Ekere K, Johnson Isaac A, Okoh JE, Emeje M. Green Preparation of Citric Acid Crosslinked Starch for Improvement of Physicochemical Properties of Cyperus Starch. Turk J Pharm Sci. 2021;18:34-43.

2. González-Rivera J, Spepi A, Ferrari C, Duce C, Longo I, Falconieri D, Piras $A$, Tinè MR. Novel configurations for a citrus waste based biorefinery: from solventless to simultaneous ultrasound and microwave assisted extraction. Green Chem. 2016;18 6482-6492.

3. Shiotsubo T, Takahashi K. Differential thermal analysis of potato starch gelatinization. Agric Biol Chem. 1984;48:9-17.

4. Chang R. Physical Chemistry for the Chemical and Biological Sciences. Sausalito; University Science Books; 2000:142-144.

5. Cowan ML, Kang J. Enthalpy-Entropy Compensation in the Melting of Human Hair. Int J Trichology. 2019;11:141-142. 